\title{
Comparative Anatomy of the Radial Sesamoid Bone in the Polar Bear (Ursus maritimus), the Brown Bear (Ursus arctos) and the Giant Panda (Ailuropoda melanoleuca)
}

\author{
Hideki ENDO, Takashi MAKITA ${ }^{1) *}$, Motoki SASAKI ${ }^{2)}$, Kazuyoshi ARISHIMA ${ }^{3)}$, Masako YAMAMOTO ${ }^{3)}$ and \\ Yoshihiro HAYASHI ${ }^{2,4)}$ \\ Department of Zoology, National Science Museum, Tokyo, 3-23-1 Hyakunin-cho, Shinjuku-ku, Tokyo 169-0073, ${ }^{\text {I)Department of }}$ \\ Veterinary Anatomy, Faculty of Agriculture, Yamaguchi University, Yamaguchi 753-8515, ${ }^{2}$ The University Museum, The University of \\ Tokyo, Bunkyo-ku, Tokyo 113-0033, ${ }^{3}$ Department of Veterinary Anatomy II, Azabu University School of Veterinary Medicine, \\ Kanagawa 229-8501, ${ }^{4}$ Department of Veterinary Anatomy, Graduate School of Agricultural and Life Sciences, The University of Tokyo, \\ Bunkyo-ku, Tokyo 113-8657, Japan
}

(Received 17 February 1999/Accepted 7 April 1999)

ABSTRACT. Since we have clarified the manipulation mechanism using the radial sesamoid (RS) in the giant panda (Ailuropoda melanoleuca), our aim in this study is to examine the position, shape and function of the RS morphologically, and to observe the attachment to the RS of the M. abductor pollicis longus and the M. opponens pollicis in the other Ursidae species. So, we focused on the carpus and manus of the polar bear (Ursus maritimus) and the brown bear (Ursus arctos) in this study. The RS was tightly articulated to the radial carpal, and could not adduct-abduct independently of the radial carpal. The M. abductor pollicis longus tendon and the $M$. opponens pollicis belly were attached to the RS, independently. In the polar bear, the deep concave and the flat surface were confirmed in attachment area for these two muscles. The morphological relationship between the RS and the M. abductor pollicis longus and the M. opponens pollicis in the two species of bears were essentially consistent with that in the giant panda. It also demonstrated that the manipulation mechanism of the giant panda has been completely based on the functional relationship between the small RS, and the $M$. abductor pollicis longus and the M. opponens pollicis in Ursidae species.-KEY wORDS: brown bear, giant panda, polar bear, radial sesamoid bone.

Many anatomists have been interested in the huge radial sesamoid bone (RS) of the giant panda (Ailuropoda melanoleuca) since the beginning of the 20th century [1, 3, $6,8,11,12]$. The giant panda uses the largely-developed RS in addition to digits to grasp food [11]. Because the giant panda taxonomically belongs to the Ursidae $[2,9,10]$, we have expected to examine the RS in the other Ursidae species morphologically. In the present study, therefore, we used the carcass of the polar bear (Ursus maritimus), the brown bear (Ursus arctos) and the giant panda, and carried out detailed observations and comparisons in the shape and size of RS, and the occurrence of RS-related muscles among these three species.

\section{MATERIALS AND METHODS}

In this study, we used the adult carcass of the polar bear, brown bear, and giant panda, that were donated to the National Science Museum, Tokyo, from Ritsurin Park Zoo (Kagawa, Japan) [7] and Ueno Zoological Park (Tokyo, Japan) (Table 1). Both the left and right forelimbs were separated from the carcass trunk and the forearms were dissected in the cold room at $-4^{\circ} \mathrm{C}$. Fixation of the carcass in formalin was avoided, so that the skeleton specimen could

* Present address: Makita, T., Azabu University School of Veterinary Medicine, Kanagawa 229-8501, Japan. be well prepared. The RS-related muscles were observed after excising the skin and subcutaneous tissues. Following the observation, the RS was removed from the forearms in the polar bear and the brown bear, and was described using the light microscope. The anatomical nomenclature was based on the monograph of Davis [3], because the nomenclature of the veterinary anatomy is not always useful in the functional anatomy of carpal region in carnivores.

\section{RESULTS}

The RS is not regular in shape in the two species of the bears (Figs. 1, 2). In the brown bear, the RS shows the longer body than that in the polar bear. In the polar bear, the muscle attachment areas are most noteworthy. The surface is smooth and flat to receive the bundles of the $M$. opponens pollicis in ulnar aspect (Fig. 1). In the polar bear, the deep concave area is discerned on radial side (Fig. 2). The tendon of M. abductor pollicis longus is inserted into the concave from radial side of the SR. In smaller RS of the brown bear, however, we cannot distinguish the insertion-surface for the M. opponens pollicis. The concave for the M. abductor pollicis longus bundles is not confirmed in radial aspect in the brown bear.

The RS is located in the radio-proximal area of the first metacarpal in the polar bear and brown bear (Figs. 3-6). The side articulating to the radial carpal (intermedioradial 
Table 1. The carcass specimen data used in this study

\begin{tabular}{llccc}
\hline Species & Museum No. & $\begin{array}{c}\text { Age } \\
\text { (years) }\end{array}$ & Sex & Donor \\
\hline Polar bear & NSMT-M31421 & $20^{*}$ & male & Ritsurin Park Zoo \\
Brown bear & NSMT-M31422 & 17 & female & Ueno Zoological Park \\
Giant panda & NSMT-M30000 & $25^{*}$ & male & Ueno Zoological Park \\
\hline
\end{tabular}

*Ages were estimated by donors in the polar bear and the giant panda.
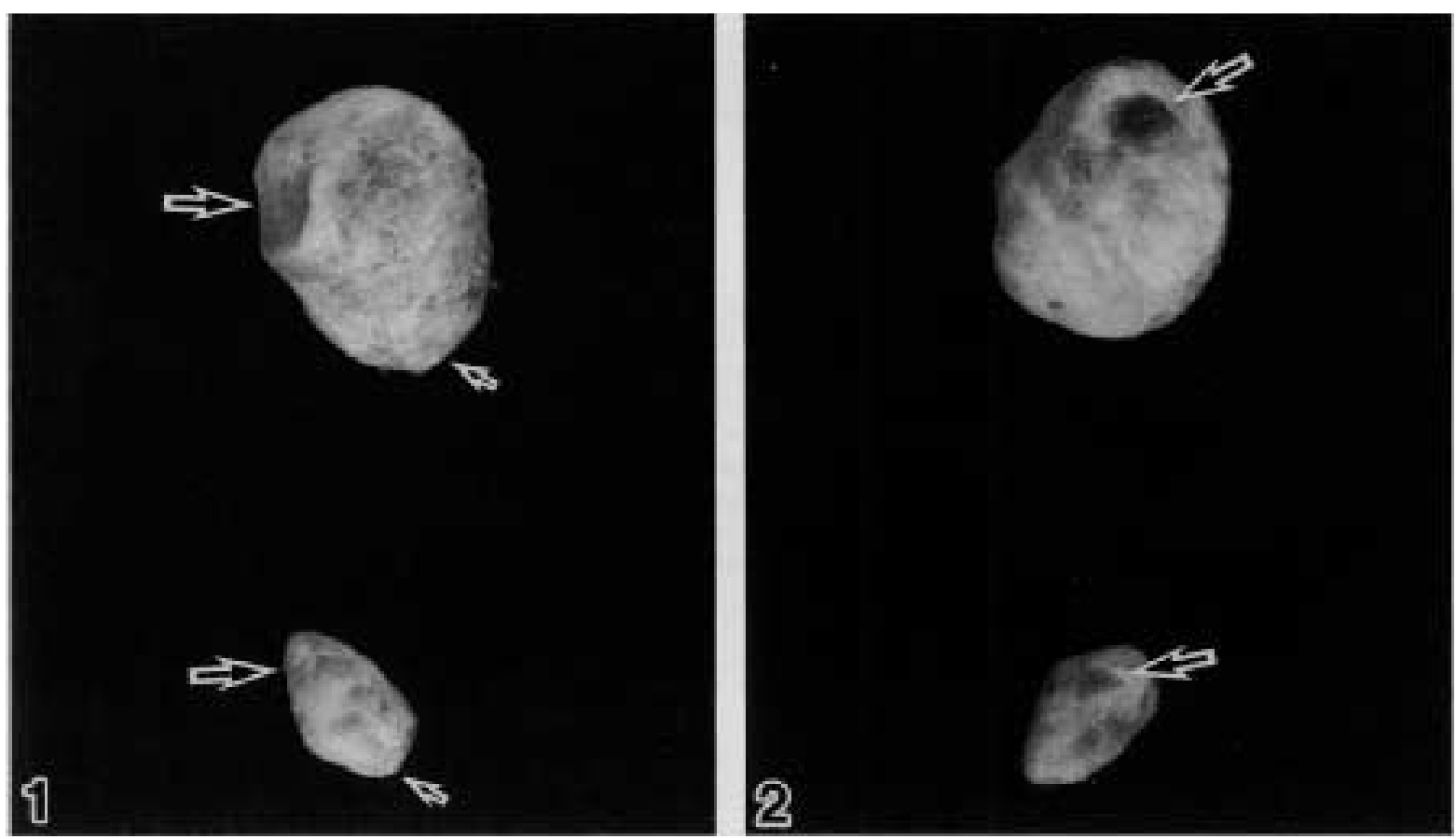

Fig. 1. Ulnar aspect of the right radial sesamoid. Upper: the polar bear. Lower: the brown bear. Small arrows indicate the articulated part with the radial carpal in each bone. The bundles of the $M$. opponens pollicis have been inserted into the flat area in the polar bear (large arrow). In the brown bear, however, we cannot obviously identify the attachment surface for the $M$. opponens pollicis (large arrow).

Fig. 2. Radial aspect of the right radial sesamoid. Upper: the polar bear. Lower: the brown bear. Articulated area with the radial carpal is located at the bottom in each bone. The M. abductor pollicis longus tendon has been attached to the deep concave in the polar bear (arrow). In the brown bear, however, we cannot obviously distinguish the concave for the M. abductor pollicis longus (arrow).

carpal or Scapholunatum) is not flat but sharp (Figs. 1, 2). The well-developed connective tissue has the RS attached to the radial carpal in these species. The connective tissue occurs from the distal surface of the radial carpal and is inserted into the whole lateral area of the RS.

The M. abductor pollicis longus arises from the radius and ulna, and sends the strong tendons to the RS and the first metacarpal (Figs. 3-6). In the polar bear and the brown bear, we observed the tendon groups running near the RS to the first metacarpal. The closest tendon to the RS attaches to the radial surface of the RS in both species (Figs. 3-6). The tendon possesses only the narrow attachment part to the RS in the brown bear (Figs. 3, 5).

The connecting length of the $M$. abductor pollicis longus tendon is $8 \mathrm{~mm}$ in the polar bear and $4 \mathrm{~mm}$ in the brown bear (Figs. 3, 5), however, after the narrow attachment, the tendons are strongly inserted into the proximal area of the first metacarpal.

The M. opponens pollicis belly is present on the palmar side of the first metacarpal (Figs. 3-6). In both polar and brown bears, some bundles of the $M$. opponens pollicis arise from the first carpal (Trapezium), and reach the ulnar side of the RS (Figs. 3, 6). The bundle is narrow and thin, however, we can distinguish its attachment to the RS on the ulnar surface in both bears. The main bundles of the $M$. opponens pollicis are inserted into the palmar area of the first metacarpal.

In the giant panda, the RS is much larger than the other species (Fig. 7). The M. abductor pollicis longus send some peculiar tendons to the radio-proximal area of the RS. The 

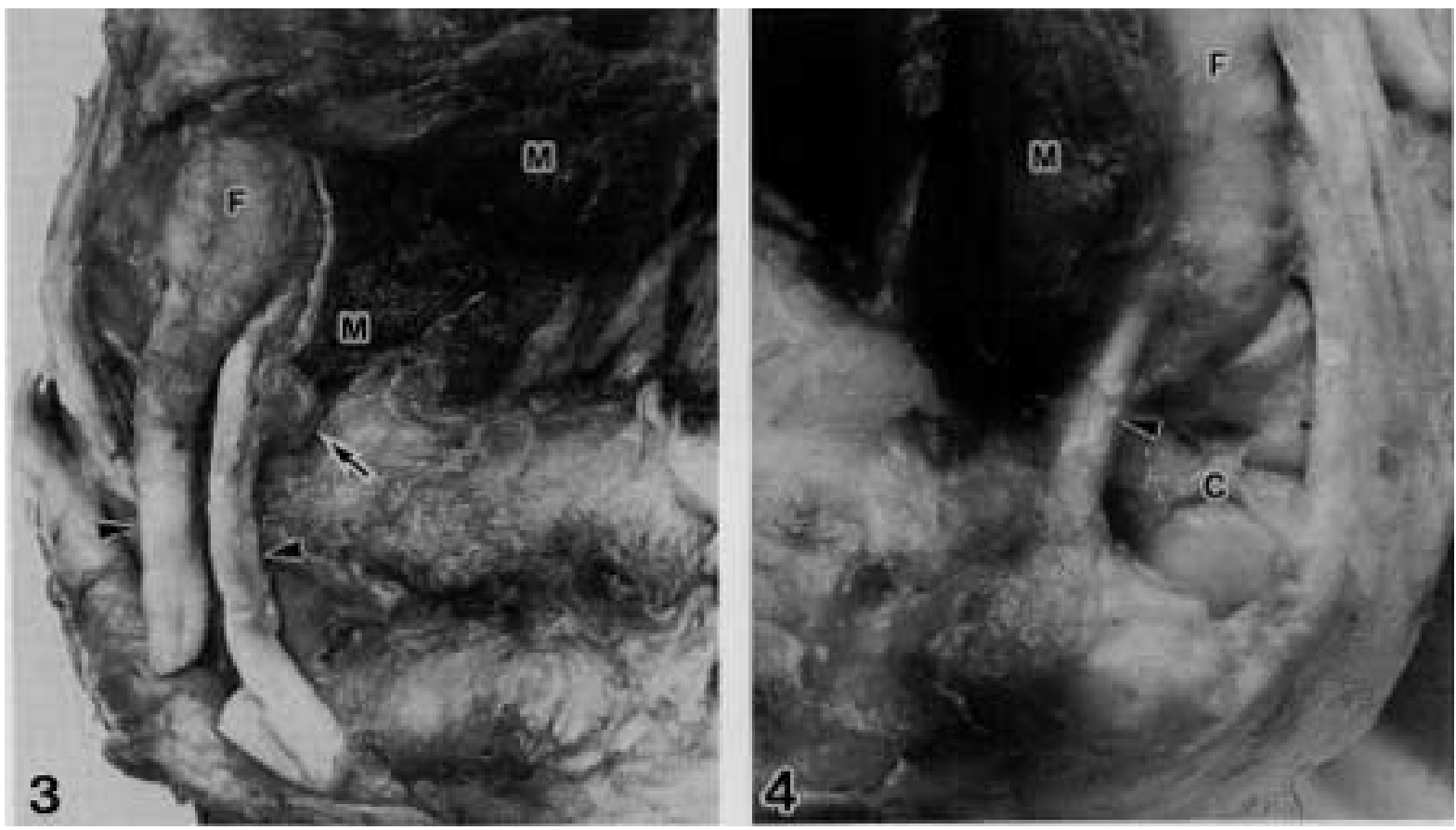

Fig. 3. Radial aspect of left carpal area of the polar bear. Distal direction at the top. The radial sesamoid (arrow) is tightly articulated to the radial carpal. The bundles of the M. opponens pollicis (M) are partially inserted into the radial sesamoid from ulnar region. One of the tendons (arrowheads) of the M. abductor pollicis longus is attached to the radial sesamoid from radial region. F, the first metacarpal.

Fig. 4. Radial aspect of right carpal area of the brown bear. Distal direction at the top. The M. opponens pollicis (M) is present in palmar portion of the first metacarpal (F). The strong tendon of the M. abductor pollicis longus (arrowhead) is inserted into the first metacarpal. C, radial carpal.

M. opponens pollicis and the M. abductor pollicis brevis are entirely attached to the ulnar side of the RS.

\section{DISCUSSION}

In the polar bear, the RS obviously possesses the attachment surfaces for the M. abductor pollicis longus tendon and the M. opponens pollicis belly, respectively. The area, into which the $M$. opponens pollicis belly is inserted, represents the flat surface in ulnar aspect, while the attachment area of the M. abductor pollicis longus tendon consists of a distinct hollow. In the brown bear, these attachment areas are not obviously seen on the bone surface. It can be explained that the polar bear possesses the larger RS than the brown bear, because the polar bear is much bigger in body size than the brown bear [10]. However, we can conclude that the attachment structure is well-developed in the RS of the polar bear as expected from the body size.

We morphologically detailed the RS of the giant panda [4], and clarified its functional significance by $3 \mathrm{D}$ analyzing techniques [5]. In the giant panda, the RS possesses the definite articulating surface to the radial carpal and the first metacarpal, while the first metacarpal has the radial sesamoid connecting process on its radial side [4]. We concluded that the RS is much more specialized in the giant panda than in other bears. We suggest that the giant panda gained the huge RS articulated to the radial carpal and the first metacarpal in the evolutionary process from an Ursidae ancestor with the smaller RS. This morphological change in the RS obviously enables the giant panda to grasp the objects in the palm. However, we [5] pointed out that the RS does not act as an active manipulator but as a part of functional unit, when the panda grasps the objects. In the present results on the two bears, the RS cannot adductabduct independently of the radial carpal as shown in the giant panda [5].

The morphological relationship between the RS and the M. abductor pollicis longus and the M. opponens pollicis in the giant panda is essentially consistent with that in two other bears. It also demonstrates that the manipulation mechanism of the giant panda has been completely based on the functional relationship between the small RS, and the M. abductor pollicis longus and the M. opponens pollicis in Ursidae species, as suggested by anatomists $[1,3,6,11$, 12].

ACKNOWLEDGEMENTS. We thank Dr. Mitsuko Masui, Dr. Masaru Saito, Dr. Yoshitaka Abe, and Dr. Etsuo Narushima and the staff of Ueno Zoological Park for providing the rare materials of the brown bear and the giant 

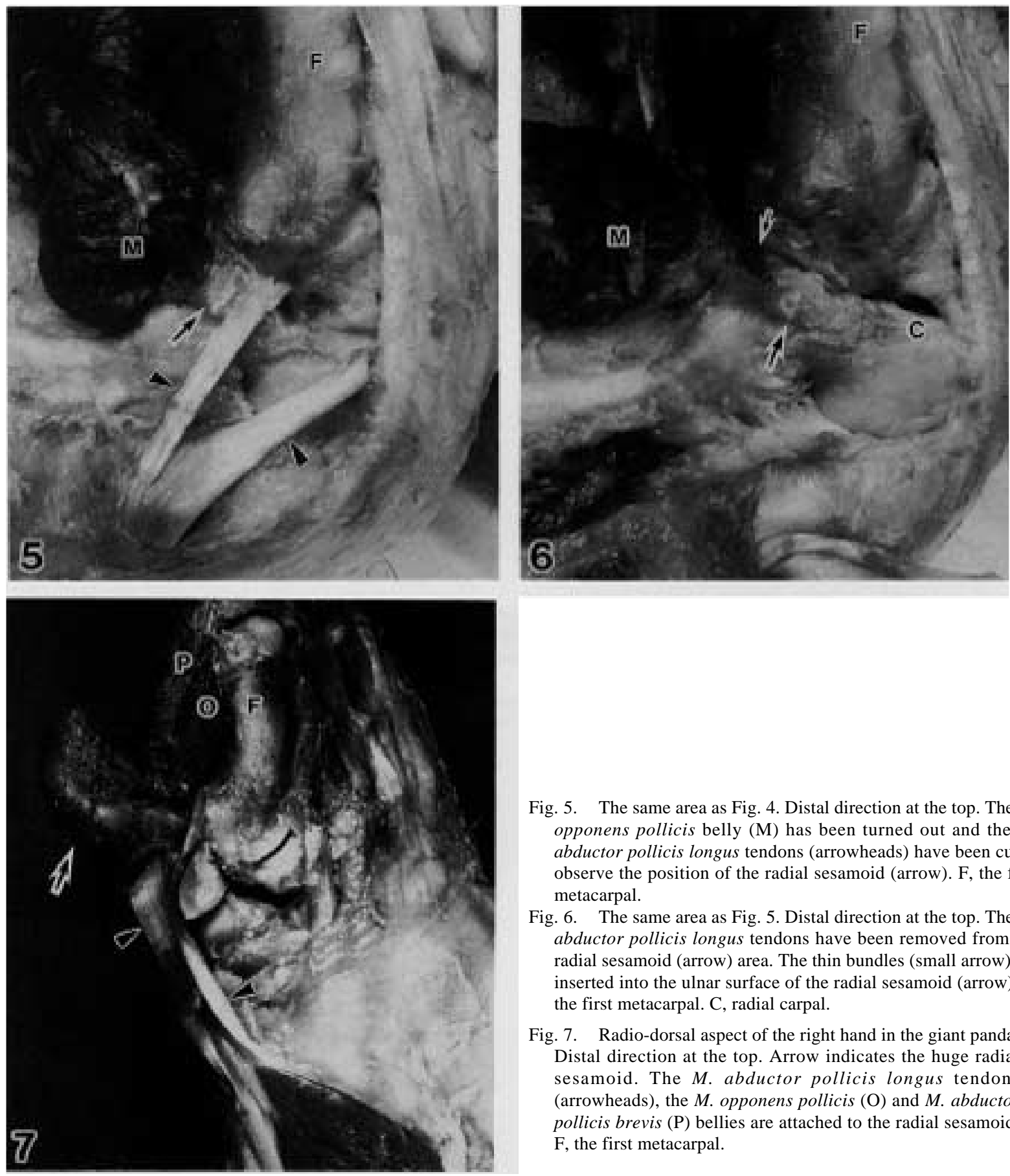

Fig. 5. The same area as Fig. 4. Distal direction at the top. The $M$. opponens pollicis belly (M) has been turned out and the $M$. abductor pollicis longus tendons (arrowheads) have been cut to observe the position of the radial sesamoid (arrow). F, the first metacarpal.

Fig. 6. The same area as Fig. 5. Distal direction at the top. The $M$. abductor pollicis longus tendons have been removed from the radial sesamoid (arrow) area. The thin bundles (small arrow) are inserted into the ulnar surface of the radial sesamoid (arrow). F, the first metacarpal. C, radial carpal.

Fig. 7. Radio-dorsal aspect of the right hand in the giant panda. Distal direction at the top. Arrow indicates the huge radial sesamoid. The M. abductor pollicis longus tendons (arrowheads), the M. opponens pollicis $(\mathrm{O})$ and M. abductor pollicis brevis $(\mathrm{P})$ bellies are attached to the radial sesamoid. $\mathrm{F}$, the first metacarpal.

panda. We wish to express our gratitude to Dr. Kazumi Kagawa (Ritsurin Park Zoo) for the donation of a carcass of the polar bear. We are also grateful to Miss Tomoko Ogoh, Miss Tomoko Yoshida and Miss Norika Yakuo of National Science Museum, Tokyo, for their encouragement of this work.

\section{REFERENCES}

1. Beijing Zoo, Beijing University, Beijing Agricultural University, Beijing Second Medical College, Beijing Natural History Museum, and Shaanxi Zoology Institute.1986. pp. 148-152. Morphology of the Giant Panda. Systematic Anatomy and Organ-Histology. Science Press, Beijing (in Chinese).

2. Corbet, G. B. and Hill, J. E. 1991. A World List of Mammalian Species, 3rd ed., Oxford University Press, Oxford. 
3. Davis, D. D. 1964. The Giant Panda. A Morphological Study of Evolutionary Mechanisms. Fieldiana Zoology Memoirs, vol. 3. Chicago Natural History Museum.

4. Endo, H., Sasaki, N., Yamagiwa, D., Uetake, Y., Kurohmaru, M. and Hayashi, Y. 1996. Functional anatomy of the radial sesamoid bone in the giant panda (Ailuropoda melanoleuca). J. Anat. 189: 587-592.

5. Endo, H., Yamagiwa, D., Hayashi, Y., Koie, H., Yamaya, Y. and Kimura, J. 1999. Role of the giant panda's 'pseudothumb'. Nature (Lond.) 397: 309-310.

6. Lankester, E. R. and Lydekker, R. 1901. On the affinities of Ailuropus melanoleucus, A. Milne-Edwards. Trans. Linn. Soc. Lond., Zool. 8: 163-171.

7. Makita, T., Koriyama, N., Namba, T., Okubo, J., Endo, H. and Kagawa, K. 1998. Gross anatomy of the lobated kidney of a male polar bear (Thalarctos maritimus). Jpn. J. Zoo.
Wildl. Med. 3: 79-82 (In Japanese with English abstract).

8. Pocock, R. I. 1939. The prehensile paw of the giant panda Nature (Lond.) 143: 206.

9. Talbot, S. T. and Shields, G. F. 1996. A phylogeny of the bears (Ursidae) inferred from complete sequence of three mitochondrial genes. Molec. Phylogenet. Evol. 5: 567-575.

10. Walker, E. P. 1983. Family Ursidae. pp. 964-977. In: Walker's Mammals of the World, vol. 2, 4th ed. Johns Hopkins University Press, Baltimore and London.

11. Wood-Jones, F. 1939. The forearm and manus of the giant panda, Ailuropoda melanoleuca, M.-Edw. with an account of the mechanism of its grasp. Proc. Zool. Soc. Lond. B 109: 113-129.

12. Wood-Jones, F. 1939. The "thumb" of the giant panda. Nature (Lond.) 143: 157. 